\title{
Direct thermal conductance measurements on suspended monocrystalline nanostructures
}

\author{
T. S. Tighe, J. M. Worlock, ${ }^{\text {a) }}$ and M. L. Roukes ${ }^{\text {b) }}$ \\ Condensed Matter Physics, California Institute of Technology 114-36, Pasadena, California 91125
}

(Received 17 December 1996; accepted for publication 21 March 1997)

\begin{abstract}
We describe and demonstrate a new class of devices that enable direct thermal conductance measurements on monocrystalline nanostructures. These are possible through our newly developed techniques for three-dimensional, successive surface nanomachining of GaAs-based heterostructures. Our methods allow the patterning of complex devices comprising electrically insulating, mesoscopic thermal conductors with separate, thermal transducers in situ. Intimate thermal contact between these elements is provided by their epitaxial registry. Low-temperature thermal conductance measurements indicate that phonon boundary scattering in these initial nanometer is scale structures is partially specular. These devices offer promise for ultrasensitive bolometry and calorimetry. (C) 1997 American Institute of Physics. [S0003-6951(97)02720-4]
\end{abstract}

Despite many intriguing electron transport phenomena discovered in semiconductor nanostructures in the last decade, little progress has been made toward elucidating energy transport involving phonons in this size regime. This is in large part due to the special fabrication required to create optimal samples for such work, they must be nanostructures with three-dimensional (3D) relief. In this letter, we describe newly developed lithographic techniques we have developed for such studies. These permit fabrication of isolated, multicomponent, 3D structures with dimensions at or below $L$ $\sim 100 \mathrm{~nm}$, i.e., smaller than the dominant phonon wavelength, $\lambda_{\mathrm{dom}}$, for $T<0.5 \mathrm{~K} .{ }^{1}$ In small structures at low temperatures, where $\lambda_{\mathrm{dom}} \gg L$, reduced dimensional phonon transport effects are expected. We demonstrate our new approach through the first direct thermal conductance measurements in nanostructures, carried out in this initial work at somewhat higher temperatures, $T>1.5 \mathrm{~K}$.

It has been possible to fabricate suspended nanostructures for some time, ${ }^{2}$ but the lack of local, nanometer scale thermal transducers has posed a serious barrier to explorations of energy transport and, specifically, the phonon thermal conductance, $K_{\mathrm{ph}}$, in mesoscopic systems. Ideally, such transducers (heat source and sensor) should be local, yet separate, entities with respect to the mesoscopic thermal conductor under investigation. In the experiments carried out to date, however, all three of these functions have typically been provided by a single entity. Generally, this has been a narrow conducting wire that is, to some degree, thermally isolated from its environment, either by suspending it at each end, ${ }^{3,4}$ or through poor coupling to the substrate..$^{5}$ Electron heating, rather than thermal conductance, is studied in these isolated wires; an applied dc current causes Joule heating of the electrons while, simultaneously, the temperature dependence of the wire's ac (low frequency) conductance provides the electron temperature. In these experiments, the suspended, conducting wire itself constitutes the thermal conductor under investigation. The underlying idea is that reduction of dimensionality of the phonon system will be reflected

\footnotetext{
${ }^{a)}$ Permanent address: Department of Physics, University of Utah, Salt Lake City, Utah.

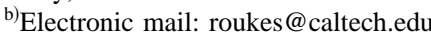

as an impairment of the electron cooling process. A very significant thermal short circuit exists along the electron gas itself, however, with the result that phonons typically carry very little heat in the temperature regime of interest [where $\left.\lambda_{\text {dom }}(T) \gg L\right]$. This "parasitic", thermalization pathway arises due to the diffusion of hot electrons down the electron gas to the colder supports; at low temperatures, only a minuscule amount of the Joule heat is transferred to the lattice by electron-phonon scattering. ${ }^{3}$ In this situation phonon heating is minimal, and thermal conductance can only be deduced after rather involved modeling is employed (with attendant assumptions about nonequilibrium scattering rates) to extract the almost negligible phononic contribution from the dominant, electronic heat loss mechanism. Additionally, such an approach is restricted to conducting wires and, thus, precludes the most general class of phonon transport investigations involving mesoscopic insulators of arbitrary geometry.

Our approach is motivated by a desire to configure nanostructures for direct thermal conductance measurements. Ideally, the simplest approach would be to thermally clamp one end of a nanostructured beam under study while providing heat, $\dot{Q}$, to the other thermally isolated end. For small heat input, to lowest order, the thermal conductance is then $K$ $=\dot{Q} / \Delta T$, where $\Delta T$ is the temperature difference between the clamped and thermally isolated ends. ${ }^{6}$ Fabrication of nanometer scale structures in such a configuration is, however, geometrically problematic. Instead, we take an alternative, equivalent approach exemplified by the device depicted in Fig. 1. This device comprises a thin, rectangular intrinsic ( $i$-) GaAs thermal reservoir suspended above the substrate by four $i$-GaAs beams. The latter constitute the thermal conductors of interest. The isolated reservoir is Joule heated by a source transducer patterned above it; the reservoir cools through the long, narrow, monocrystalline $i$-GaAs bridges that suspend it. Measurement of an elevated reservoir temperature, arising in response to this heat input, is achieved using a second, and separate, local sensor transducer. This allows direct measurement of the parallel thermal conductance of the four nanometer-scale support beams.

This new approach is possible through the nanofabrication techniques, which we describe below. ${ }^{7}$ Our approach 

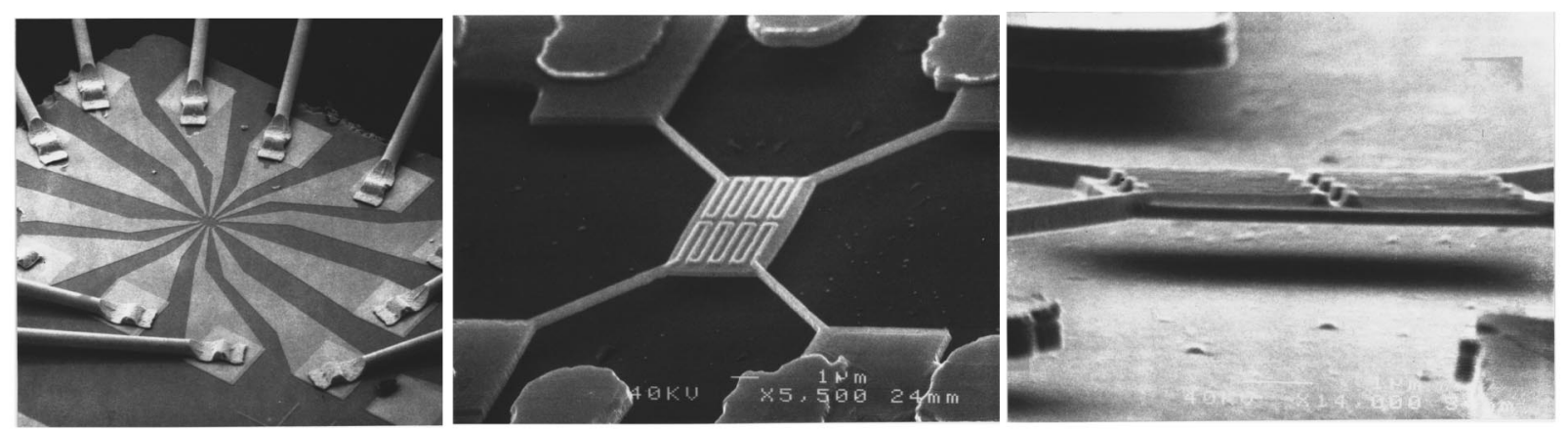

FIG. 1. Suspended, monocrystalline device for mesoscopic thermal conductance measurements. (Left) overall view of a $\sim 1$ mm ${ }^{2}$ twelve-lead device, with external, $25 \mu \mathrm{m}$ Au wirebonds. "Fingers" within a thin Au film lead frame connect wirebond pads to the central region of the device. (Center) enlarged view of central region showing a semi-insulating $i$-GaAs reservoir $\left(\sim 3 \mu \mathrm{m}^{2}\right)$ suspended by four, $5.5 \mu \mathrm{m}$ long $i$-GaAs bridges (cross section $\sim 200 \mathrm{~nm} \times 300 \mathrm{~nm})$. An integral pair of meandering $n+\mathrm{GaAs}$ conductors $(\sim 100 \mathrm{~nm}$ linewidth), in epitaxial registry with the underlying $i$-GaAs reservoir, constitute transducers for the measurement. (Right) edge view of the device; it is $\sim 300 \mathrm{~nm}$ thick and is suspended about $1 \mu \mathrm{m}$ above the substrate.

yields devices that are essentially monocrystalline in their totality; through epitaxy, the thermal conductors under investigation and their associated transducers are intimately coupled, are all in atomic registry. Our process yields excellent dimensional control of the different layers, limited solely by the resolution of electron-beam (e-beam) lithography in the lateral directions, and by the epitaxial growth process in the vertical direction (which provides thickness control at the monolayer level).

Our current devices are patterned from GaAs heterostructures comprising three epilayers grown by molecular beam epitaxy upon an undoped, semi-insulating (i) GaAs substrate. Heavily $\mathrm{Si}$ doped, $n+\mathrm{GaAs} \quad\left(N_{D}=2\right.$ $\times 10^{18} \mathrm{~cm}^{-3}$ ) forms the topmost conducting structural layer; the electrical transducers are subsequently patterned from this. The second (semi-) insulating structural layer consists of undoped $i$-GaAs; from this are formed the isolated reservoir and the thermal conductors to be studied. Beneath this is the third, AlAs sacrificial layer that ultimately enables suspension of the device. For the structure of Fig. 1, these layer thicknesses were $150 \mathrm{~nm}, 300 \mathrm{~nm}$, and $1 \mu \mathrm{m}$, respectively.

Fabrication of these devices involves two separate electron-beam lithography steps to define, first, the $n+$ electrical conductors and, second, the electrically insulating reservoir and beams. In the first step, a Ni mask is rf sputter deposited and patterned by e-beam lithography. An anisotropic, vertical, chemically assisted ion beam etching (CAIBE) step $^{8}$ is then carefully timed to remove all of the $n+$ layer except that beneath the $\mathrm{Ni}$ mask. The etch rate of the Ni mask is two orders of magnitude slower than that of GaAs. In the second step, e-beam lithography is employed to align a second Ni mask, which serves to define the isolated reservoirs, to the previously patterned $n+$ transducers. A subsequent CAIBE etch step is then used to cut vertically downward through all layers to the substrate. The sample is then subjected to two chemically selective etchants that remove solely the sacrificial material and, finally, the Ni mask.

Measurement of these devices begins with calibration of the temperature-dependent resistance, $R(T)$, of the sensor by standard four-probe ac resistance measurements. Separate measurements indicate that a $200 \mathrm{pA}$ sensing current does not measurably self-heat the transducer. $R(T)$ varies by $\sim 5 \%$ over the range from $1.5-6 \mathrm{~K}$ due to localization and interaction effects (Fig. 2). ${ }^{9-11}$ The meandering pattern of the $n+\mathrm{GaAs}$ conductors posits most of the line length above the suspended reservoir. From the transducers, $n+$ leads on top of each bridge provide electrical contact to wirebond pads. The $n+$ transducers, the only electrically conducting entities within the device, are nominally identical; their $R(T)$ differ by only $\sim 5 \%$ (Fig. 2 ).

The phonon thermal conductance is obtained by heating the source transducer with a small dc current while monitor$\operatorname{ing} T_{s}$, the electron temperature of the unbiased sensor. Over most of the experimental range, $T_{s}$ corresponds closely to $T_{\mathrm{ph}}$, the lattice temperature of the isolated reservoir. ${ }^{11}$ In Fig. 3 (a) we display the thermal conductance, $K_{\mathrm{ph}}=\dot{Q} / \Delta T$, versus $T_{s}$, where $\Delta T$ is the difference between $T_{s}$ at the base temperature $(\dot{Q}=0)$ and for $\dot{Q}$ finite. In Fig. 3(b), we plot the effective mean-free path, estimated as $\Lambda_{\text {eff }}$ $=3 K_{\mathrm{ph}} \ell /\left(4 C_{\mathrm{ph}} c_{\mathrm{av}} A\right)$ for the four bridges in parallel. Here, $\ell$ and $A$ are the bridge length and cross-sectional area, $c_{\mathrm{av}}$ is the angle and polarization averaged phonon velocity, and $C_{\mathrm{ph}}=\left(12 \pi^{4} n k_{B} / 5\right)\left(T / \Theta_{D}\right)^{3}$ is the Debye heat capacity, a good approximation for $T \ll \Theta_{D}$. For GaAs, the molecular density, $n$, is $2.2 \times 10^{22} \mathrm{~cm}^{-3}$ and the Debye temperature, $\Theta_{D}$, is $345 \mathrm{~K}$. As shown, we deduce $\Lambda_{\text {eff }} \sim 0.51 \mu \mathrm{m}$ in this manner for temperatures above $\sim 3 \mathrm{~K}$. A rough indication of the surface quality of our nanofabricated bridge is given by the specularity parameter $p=\left[\left(\Lambda_{\text {eff }} / \Lambda_{0}\right)-1\right] /\left[\left(\Lambda_{\text {eff }} / \Lambda_{0}\right)+1\right]$; for mirrorlike scattering $p=1 .{ }^{12}$ Here, $\Lambda_{0}$ is the mean-free

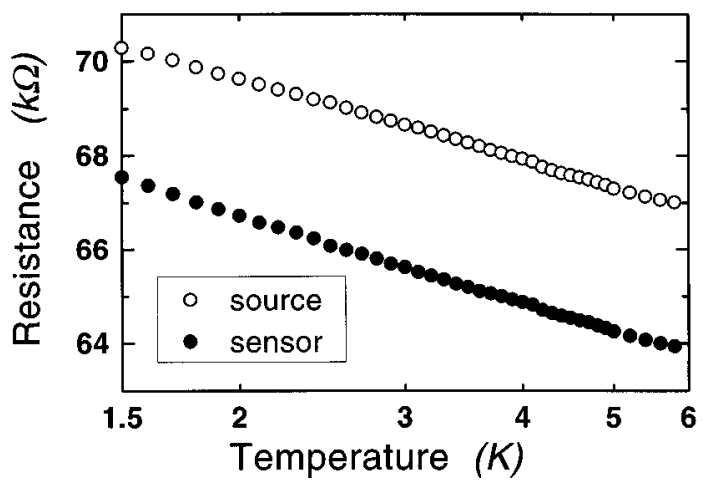

FIG. 2. Temperature-dependent resistance of source and sensor transducers between 1.5 and $6 \mathrm{~K}$. 


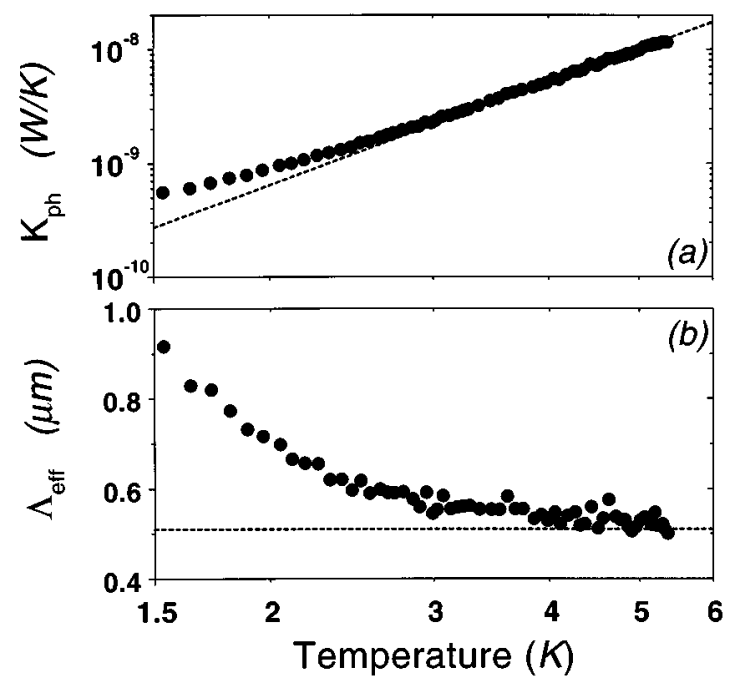

FIG. 3. (a) Phononic thermal conductance from the parallel measurement of four $200 \mathrm{~nm} \times 300 \mathrm{~nm}$ GaAs bridges. The dashed line represents the predictions of the Debye model for a fixed boundary-limited mean-free path of $0.51 \mu \mathrm{m}$. (b) Effective mean-free path deduced from the experimental data.

path in the diffuse scattering limit, $p=0$. For a rectangular cross section of side $d, \Lambda_{0} \sim 1.12 d$, hence, $\left(\Lambda_{\text {eff }} / \Lambda_{0}\right) \sim 1.86$ for our samples. ${ }^{13}$ Above $3 \mathrm{~K}$, we thereby deduce $p \sim 0.3$, indicating that phonon boundary scattering is partially specular in these narrow channels, i.e., ballistic phonon transport decays after $1 /(1-p) \sim 2$ surface reflections. In future work, we shall explore variations of these fabrication methods to minimize diffuse boundary scattering. The rising $\Lambda_{\text {eff }}$ at low temperatures in these data is tantalizing; an increase is expected when $\lambda_{\text {dom }}$ exceeds the correlation length for surface roughness. Here, however, this rise appears to be largely due to extraneous thermal coupling between the sensor and supports via the $n+$ leads; separate experiments indicate this becomes increasingly important for $T<2.5 \mathrm{~K}$. ${ }^{11}$ In future sample configurations, this undesired thermal path will be eliminated.

We have described a new approach for the successive surface nanomachining of monocrystalline GaAs heterostructures. This enables the patterning of devices comprising insulating thermal conductors, with local thermal transducers in situ; with these we have demonstrated direct thermal conductance measurements on suspended nanostructures. Future work will be carried out at $\mathrm{mK}$ temperatures, where mesoscopic phenomena should be manifested. We note, also, that these devices have extremely small heat capacities at low temperatures. In current work, we are exploring their potential for ultrasensitive bolometry and calorimetry.

The authors thank Axel Scherer and Larry Schiavone for important early contributions, and Leigh Florez, Jim Harbison, and Henry Lee for providing heterostructures critical to these efforts.

${ }^{1}$ We approximate the acoustic subband separations, assuming free boundaries and linear dispersion, as $E_{n}=2 n \pi \hbar c_{s} / L$, where $c_{s}$ is a longitudinal or transverse sound velocity. To sweep the Planck distribution peak $\left(E_{\text {peak }} \sim 2.82 k_{B} T\right)$ below the second subband $(n=1)$ requires $2 \pi \hbar c_{s} /\left(2.82 k_{B} T L\right) \equiv \lambda_{\mathrm{dom}} / L \gg 1$.

${ }^{2}$ See, e.g., C. G. Smith, H. Ahmed, and M. N. Wybourne, J. Vac. Sci. Technol. B 5, 314 (1987).

${ }^{3}$ A. Potts, M. J. Kelly, D. B. Hasko, J. R. A. Cleaver, H. Ahmed, D. A. Ritchie, J. E. F. Frost, and G. A. C. Jones, Semicond. Sci. Technol. 7, B231 (1992).

${ }^{4}$ K. Yoh, A. Nishida, H. Kawahara, S. Izumiya, and M. Inoue, Semicond. Sci. Technol. 9, 961 (1994).

${ }^{5}$ J. Seyler and M. N. Wybourne, Phys. Rev. Lett. 69, 1427 (1992).

${ }^{6}$ In this simple discussion, we ignore phonon scattering at the transducers which complicates the results when phonon boundary specularity is large. See, e.g., T. Klitsner, J. E. Van Cleve, H. E. Fischer, and R. O. Pohl, Phys. Rev. B 38, 7576 (1988).

${ }^{7}$ For related work regarding GaAs surface micromachining see, e.g., K. Hjort, J. Söderkvist, and J. A. Schweitz, J. Micromech. Microeng. 4, 1 (1994); K. Fricke, Sens. Actuators A 45, 91 (1994); Y. Uenishi, H. Tanaka, and H. Ukita, IEEE Trans. Electron Devices 41, 1778 (1994).

${ }^{8}$ M. Geis, S. W. Pang, N. E. Efremow, G. D. Johnson, and W. D. Goodhue, in Handbook of Ion Beam Processing Technology, edited by J. J. Cuomo, S. M. Rossnagel, and H. R. Kaufman (Noyes, New Jersey, 1989), p. 219.

${ }^{9}$ C. Kurdak, A. M. Chang, A. Chin, and T. Y. Chang, Phys. Rev. B 46, 6846 (1992)

${ }^{10}$ B. L. Al'tshuler and A. G. Aronov, Pis'ma Zh. Eksp. Teor. Fiz. 33, 515 (1981) [JETP Lett. 33, 499 (1981)].

${ }^{11}$ T. S. Tighe, J. M. Worlock, and M. L. Roukes (unpublished).

${ }^{12}$ J. M. Ziman, Electrons and Phonons (Oxford University Press, Oxford, 1979), Chap. XI

${ }^{13} \mathrm{We}$ use the geometric mean of the cross-sectional dimensions: $L$ $\sim 0.300 \mu \mathrm{m}$. 